\title{
$H$-Theorem for a Linear Kinetic Theory
}

\author{
J. Bławzdziewicz, ${ }^{1,2}$ B. Cichocki, ${ }^{3,4}$ and H. van Beijeren ${ }^{1}$
}

Received December 21, 1990; final July 22, 1991

\begin{abstract}
A strong $H$-theorem is proved for the approximate linear kinetic theory of Blawzdziewicz and Cichocki, obtained by truncating a transformed hierarchy of evolution equations. For an $i$ th truncation we define an entropy functional that is strictly increasing in time, unless the $i$ th reduced distribution function depends on position coordinates only. It also follows that the only stationary solution of the linear kinetic theory is the equilibrium solution. In addition, we show that the usual symmetry properties of equilibrium time correlation functions are preserved by the approximate kinetic theory under consideration.
\end{abstract}

KEY WORDS: $H$-theorem; kinetic theory; hard spheres; dense fluids.

\section{INTRODUCTION}

In kinetic theory, the possibility of proving $H$-theorems for the kinetic equations studied is always considered a very desirable property. If such a theorem holds, this implies the approach of the system, as described by the kinetic equations under consideration, toward equilibrium, even though the $H$-theorem alone is not sufficient to prove that indeed the equilibrium state is reached in the long-time limit. In addition, an $H$-theorem provides us with an $H$-function, the negative of which can be interpreted as the entropy of the system, even if it is far removed from equilibrium.

For kinetic equations that can be derived by the principle of maximization of entropy an $H$-theorem can be proved. Examples of such

\footnotetext{
${ }^{1}$ Institute for Theoretical Physics, University of Utrecht, 3508 TA Utrecht, The Netherlands.

${ }^{2}$ On leave of absence from Institute of Physics, Szczecin University, Wielkopolska 15, Szczecin, Poland.

${ }^{3}$ Institute of Theoretical Physics, Warsaw University, Hoża 69, 00681 Warsaw, Poland.

${ }^{4}$ Institute of Fundamental Technological Research, Polish Academy of Sciences, Świętokrzyska 21, 00-049 Warsaw, Poland.
} 
equations include the revised Enskog equation ${ }^{(1)}$ and its generalization to a square-well fluid without ${ }^{(2)}$ or with $^{(3)}$ a differentiable tail.

The underlying idea is the following ${ }^{(4)}$ : Let $\left[f_{\alpha}\right]$ be a set of functionals of the phase space variables $\Gamma=\left[N, \mathbf{x}_{1}, \mathbf{x}_{2}, \ldots, \mathbf{x}_{N}\right]$, where $N$ is the number of particles and $\mathbf{x}_{i}=\mathbf{r}_{i}, \mathbf{v}_{i}$ is the phase of particle $i$, such that $\left[f_{\alpha}\right]$ constitutes the set of independent variables for the kinetic equations to be derived. For example: for the revised Enskog equation $\left.\left[f_{x}\right]\right]$ is the one-particle distribution function

$$
F_{1}(\mathbf{x} ; t)=\left\langle\sum_{i=1}^{N} \delta\left(\mathbf{x}-\mathbf{x}_{i}\right)\right\rangle_{\rho(t)}
$$

where

$$
\langle A(\Gamma)\rangle_{\rho(t)}=\int d \Gamma A(\Gamma) \rho(\Gamma ; t)
$$

denotes the average of a quantity $A(\Gamma)$ over the time-dependent phase-space density $\rho(\Gamma ; t)$ and

$$
\int d \Gamma=\sum_{N=0}^{\infty} \int d \mathbf{x}_{1} \cdots d \mathbf{x}_{N}
$$

In the case of the revised Enskog equation, the subscript $\alpha$ represents the continuous set of values taken by the phase space variable $\mathbf{x}$. Now for a given set $\left[f_{\alpha}\right]$ one may construct the phase space density $\rho_{\max }\left(\Gamma,\left[f_{\alpha}\right]\right)$ that maximizes the entropy

$$
S[\rho]=-k_{\mathbf{B}} \int d \Gamma \rho(\Gamma) \ln \left[N ! h^{3 N} \rho(\Gamma)\right]
$$

with $k_{B}$ and $h$ Boltzmann's and Planck's constants, respectively, under the constraints that $\rho_{\max }$ reproduces the given set of values of $\left[f_{x}\right]$ correctly. Accordingly one assigns to the set $\left[f_{\alpha}\right]$ the entropy

$$
S\left[f_{\alpha}\right]=S\left[\rho_{\max }\left(\Gamma,\left[f_{\alpha}\right]\right)\right]
$$

Obviously

$$
S\left[f_{\alpha}\right] \geqslant S[\rho]
$$

if $\rho$ satisfies the constraints on $\left[f_{\alpha}\right]$. Now, if initially $\rho(\Gamma, 0)=\rho_{\max }(\Gamma, 0)$, the kinetic equations derived by the principle of maximization of entropy are exact at the initial time $t=0$. As a consequence, the exact time 
evolution of the ensemble and the kinetic equations for $\left[f_{\alpha}\right]$ yield the same set of values for $\left[f_{x}\right]$ at $t=0^{+}$, an infinitesimal time beyond $t=0$. As a result of Liouville's theorem, the entropy according to (1.1) is invariant in time, hence, as a consequence of (1.6),

$$
S\left[f_{x}, 0^{+}\right] \geqslant S\left[f_{\alpha}, 0\right] .
$$

As this holds for any choice of $\left[f_{\alpha}\right]$, and as the kinetic equations, determining the time evolution of $\left[f_{\alpha}\right]$ are determined by $\left[f_{\alpha}\right]$ alone, ${ }^{5}$ it follows immediately that the entropy (1.5) is a nondecreasing function of time. Proving that it actually is an increasing function is a technical problem that has to be considered from case to case and has been solved only for some special choices of $\left[f_{\alpha}\right]$. For the kinetic equations mentioned before, proofs of such strong $H$-theorems were given in refs. 2-6.

Bławzdziewicz and Cichocki ${ }^{(7,8)}$ obtained a sequence of linearized kinetic theories (LKT) for a hard-sphere (HS) system close to equilibrium by transforming the BBGKY hierarchy equations into a hierarchy of equations for a set of certain distributions possessing strong cluster properties. The full set of distributions is equivalent to the set of ordinary reduced distribution functions; the two sets can be mapped upon each other in a unique way. The collisional operators occurring in the new hierarchy equations are renormalized by static correlation functions. As a consequence of this, truncations of the new hierarchies are better suited to describe systems at high densities than truncations of the usual BBGKY hierarchy. Specifically, setting all $j$-particle reduced distribution functions with $j>i$ equal to zero yields the $i$ th-order LKT of Bławzdziewicz and Cichocki. In particular, the first-order LKT is equivalent to the linearized revised Enskog equation (LREE). ${ }^{(7,8)}$

In terms of the reduced distribution functions an entropy can be defined, which coincides with (1.4) if the latter is expanded through quadratic order in deviations of $\rho$ from equilibrium. This entropy can be shown to be a nonpositive functional of the reduced distribution functions. The $i$ th-order LKT gives rise to a truncated entropy to which only the reduced distribution functions of orders $\leqslant i$ contribute. For this truncated entropy an $H$-theorem follows in a very similar way as in the case of the kinetic equations derived by maximization of entropy:

Suppose the truncation is exact at the initial time $t=0$, so that the full entropy coincides with the truncated entropy. The exact time evolution leaves the full entropy unchanged, but between $t=0$ and $t=0^{+}$it generates

\footnotetext{
${ }^{5}$ This is of course an approximation, which is not satisfied if one follows the exact time evolution of the ensemble.
} 
an infinitesimal nonpositive contribution to the entropy, resulting from the infinitesimally nonzero $(i+1)$ th reduced distribution function at $t=0^{+}$. In the truncated entropy considered in the $i$ th-order LKT the latter contribution is lacking, but the contributions from the reduced distribution functions of orders 1 through $i$ are the same as under the exact evolution equations. Hence the truncated entropy at $t=0^{+}$is $\geqslant$the exact entropy at this time, which in turn is the same as the exact and the truncated entropy at $t=0$. So indeed it follows that in LKT entropy is a nondecreasing function of time.

In fact it can be proven that the truncated entropy is always strictly increasing, unless the $i$ th-order reduced distribution function does not depend on velocities. Hence, it follows that the only stationary solution of LKT is the equilibrium solution, where all reduced distribution functions vanish.

This paper is organized in the following way: In Sections 2-4 we briefly recall the LKT of Blawzdziewicz and Cichocki. In Section 5 we derive the $H$-theorem. In Section 6 we demonstrate some useful symmetry properties of LKT, when applied to the calculation of equilibrium time correlation functions. In Section 7 we discuss our results. Some technical details have been deferred to appendices.

\section{BASIC DEFINITIONS}

The time evolution of a system of identical hard spheres of diameter $\sigma$ can be conveniently described in terms of the binary-collision operators $T_{ \pm}$and $\bar{T}_{ \pm}$, defined by the equations ${ }^{(9,10)}$

$$
T_{ \pm}(i j)=\delta\left(r_{i j}-\sigma^{+}\right)\left|\mathbf{v}_{i j} \cdot \hat{\mathbf{r}}_{i j}\right| \theta\left(\mp \mathbf{v}_{i j} \cdot \hat{\mathbf{r}}_{i j}\right)[\hat{b}(i j)-1]
$$

and

$$
\bar{T}_{ \pm}(i j)=\delta\left(r_{i j}-\sigma^{+}\right)\left|\mathbf{v}_{i j} \cdot \hat{\mathbf{r}}_{i j}\right|\left[\theta\left(\mp \mathbf{v}_{i j} \cdot \hat{\mathbf{r}}_{i j}\right) \hat{b}(i j)-\theta\left( \pm \mathbf{v}_{i j} \cdot \mathbf{r}_{i j}\right)\right]
$$

where $\theta$ is the unit step function, $\mathbf{v}_{i j}=\mathbf{v}_{i}-\mathbf{v}_{j}, \hat{\mathbf{r}}_{i j}=\mathbf{r}_{i j} / r_{i j}$, and the operator $\hat{b}(i j)$ transforms the velocities according to the collision laws

$$
\begin{aligned}
& \hat{b}(i j) \mathbf{v}_{i}=\mathbf{v}_{i}^{\prime}=\mathbf{v}_{i}-\left(\mathbf{v}_{i j} \cdot \hat{\mathbf{r}}_{i j}\right) \hat{\mathbf{r}}_{i j} \\
& \hat{b}(i j) \mathbf{v}_{j}=\mathbf{v}_{j}^{\prime}=\mathbf{v}_{j}+\left(\mathbf{v}_{i j} \cdot \hat{\mathbf{r}}_{i j}\right) \hat{\mathbf{r}}_{i j} \\
& \hat{b}(i j) \mathbf{v}_{k}=\mathbf{v}_{k}, \quad k \neq i, j
\end{aligned}
$$

To uniquely define the result of the action of the $T$-operators upon functions which are discontinuous at the collision surface, we have introduced the Dirac $\delta$-function taken at $r_{i j}=\sigma^{+}$, where $\sigma^{+}=\sigma+|\varepsilon|,|\varepsilon| \rightarrow 0$. 
We find this more convenient than the insertion of infinitesimal freestreaming operators, which has been done in many papers. ${ }^{(9-11)}$ The $T$ - and $\bar{T}$-operators are related to each other by the conjugation relation

$$
\int d \mathbf{v}_{i} \int d \mathbf{v}_{j} f(\Gamma) T_{ \pm}(i j) g(\Gamma)=\int d \mathbf{v}_{i} \int d \mathbf{v}_{j} g(\Gamma) \bar{T}_{\mp}(i j) f(\Gamma)
$$

where $f$ and $g$ are arbitrary functions. The $T$-operators with the subscript plus and minus are used to describe the evolution forward and backward in time, respectively.

In the thermodynamic limit, one can completely describe nonequilibrium states of a macroscopic system by the set of reduced distribution functions $F_{l}, l \geqslant 1$. The reduced distribution functions are defined by the formulas

$$
F_{l}([l] ; t)=\lim _{\infty}\langle f([l] ; \Gamma)\rangle_{\rho(t)}
$$

where $\lim _{\infty}$ denotes the thermodynamic limit, $[l]=\left(\mathbf{x}_{1}, \ldots, \mathbf{x}_{l}\right)$ and

$$
f([l] ; t)=\sum_{[l(\Gamma)]} \delta([l]-[l(\Gamma)])
$$

In (2.6) the summation runs over all sequences $[I(\Gamma)]$ of $l$ different particle phases included in $\Gamma$ and

$$
\delta\left([l]-\left[l^{\prime}\right]\right)=\delta\left(\mathbf{x}_{1}-\mathbf{x}_{1}^{\prime}\right) \cdots \delta\left(\mathbf{x}_{l}-\mathbf{x}_{l}^{\prime}\right)
$$

The time evolution of the reduced distribution functions is governed by the BBGKY hierarchy. For a hard-sphere system, for positive times, the hierarchy has the form ${ }^{(10)}$

$$
\begin{aligned}
\left\{\partial_{t}\right. & \left.+\hat{L}_{0, l}-\sum_{i>j=1}^{l} \bar{T}_{-}(i j)\right\} F_{l}([l] ; t) \\
& =\sum_{i=1}^{l} \int d \mathbf{x}_{l+1} \bar{T}_{-}(i, l+1) F_{l+1}([l+1] ; t), \quad l=1,2, \ldots
\end{aligned}
$$

where

$$
\hat{L}_{0, l}=\sum_{i=1}^{l} \hat{L}_{0}(i)
$$

and

$$
\hat{L}_{0}(i)=\mathbf{v}_{i} \cdot \frac{\partial}{\partial \mathbf{r}_{i}}
$$


is the one-particle Liouville operator. The BBGKY hierarchy (2.8), with binary collisions described in terms of the operators $\bar{T}_{-}$, should be used only for times $t>0$; for negative times, $t<0$, one should replace the operators $\bar{T}_{-}$with the respective operators $-\bar{T}_{+}$, acting on the same pairs of variables.

Consider now a system displaced slightly from equilibrium. The only way to take this information into account on the level of the BBGKY hierarchy equations is via the initial conditions for $F_{k}$; the structure of the hierarchy remains unchanged. However, one can expect that near equilibrium closure of the hierarchy ought to be possible, yielding kinetic equations that provide a good approximate description of the time evolution of the system. The Enskog equation is a well-known example of such a kinetic equation in the case of hard spheres.

A general formalism of an LKT leading to a description of the evolution of an HS system in terms of binary collisions renormalized by equilibrium correlations has been proposed in refs. I and II. ${ }^{(7,8)}$ Such a renormalization of collisions is a generalization of the idea that goes into the Enskog equation. It has been shown that the renormalization can be achieved by an appropriate choice of a set of functions representing the state of a system under consideration. The new set of functions is related to the reduced distribution functions by a linear transformation. We now recall the main results of these papers. We follow here the elegant version of the formalism introduced in ref. II.

We start our analysis, in the following section, by considering the structure of the reduced distribution functions.

\section{STRUCTURE OF REDUCED DISTRIBUTION FUNCTIONS IN LKT}

LKT describes the evolution of a nonequilibrium system in such states that the reduced distribution functions can be derived, in the thermodynamic limit, from a probability density $\rho$ of the following form:

$$
\rho(\Gamma ; t)=\rho^{\mathrm{eq}}(\Gamma)[1+\psi(\Gamma ; t)-\langle\psi(\Gamma ; t)\rangle], \quad t \geqslant 0
$$

with

$$
\psi(\Gamma ; t)=\sum_{i=1}^{N} h_{1}\left(\mathbf{x}_{i} ; t\right)+\sum_{i>j=1}^{N} h_{2}\left(\mathbf{x}_{i}, \mathbf{x}_{j} ; t\right)+\sum_{i>j>k=1}^{N} h_{3}\left(\mathbf{x}_{i}, \mathbf{x}_{j}, \mathbf{x}_{k} ; t\right)+\cdots
$$

where $\rho^{\mathrm{eq}}(\Gamma)$ is the grand-canonical equilibrium distribution function. It is assumed that the functions $h_{l}([l])$, where $l \geqslant 1$, are symmetric functions of 
particle phases that vanish sufficiently fast when the distance between any pair of particles $1, \ldots, l$ tends to infinity. This last property is called the group, or cluster property of $h_{l}$, and decomposition (3.2) the linear cluster decomposition. The group property uniquely defines the decomposition (3.2) of a function $\psi$.

In many important problems concerning the behavior of a system close to equilibrium one finds the density $\rho$ in the form (3.1)-(3.2) at $t=0$. Examples of such problems include calculation of equilibrium time-correlation functions, such as those appearing in the Green-Kubo formulas for transport coefficients. One can demonstrate, by analyzing the binary-collision expansion of the evolution operator, that for systems with a short-range interparticle potential this form of the density $\rho$ can be extended for $t>0$. A formal proof of this fact has been described in ref. I. A proof, formulated in a different language than we use here, for a hard-sphere system, can be found in Chapter 5 of ref. 11.

To describe in a compact way the consequences of assumption (3.1)(3.2), it is convenient to introduce a general notation. We introduce a linear space of column vectors $\psi=\left\{\psi_{i} ; i=1,2, \ldots\right\}$ with infinite number of components, where the $i$ th component $\psi_{i}([i])$ is a symmetric function of $i$ particle phases. The scalar product of two vectors $\psi$ and $\phi$ is defined by the equations

$$
(\psi, \phi)=\sum_{i=1}^{\infty}\left(\psi_{i}, \phi_{i}\right)
$$

and

$$
\left(\psi_{i}, \phi_{i}\right)=\frac{1}{i !} \int d[\mathbf{i}] \psi_{i}^{*}([\mathbf{i}]) \phi_{i}([\mathbf{i}])
$$

where $d[\mathbf{i}]=d \mathbf{x}_{1} \cdots d \mathbf{x}_{i}$ and the asterisk denotes complex conjugation.

Next, we introduce linear operators which transform the linear space into itself. Such an operator $\hat{A}$ acting on a vector $\psi$ gives as result the vector $\hat{A} \psi$ with components

$$
(\hat{A} \psi)_{i}=\sum_{j=1}^{\infty} \hat{A}_{i j} \psi_{j}, \quad i=1,2, \ldots
$$

In our considerations the matrix elements $\hat{A}_{i j}$ are linear integral operators, defined by the following expression:

$$
\left(\hat{A}_{i j} \psi_{j}\right)([\mathbf{i}])=\frac{1}{j !} \int d\left[\mathbf{j}^{\prime}\right] A_{i j}\left([\mathbf{i}] \mid\left[\mathbf{j}^{\prime}\right]\right) \psi_{j}\left(\left[\mathbf{j}^{\prime}\right]\right)
$$

Here $A_{i j}\left([\mathbf{i}] \mid\left[\mathrm{j}^{\prime}\right]\right)$ is an integral kernel. 
The identity operator $\hat{l}$, acting in the linear space defined above, has the following structure:

$$
\hat{I}_{i j}=\delta_{i j} \hat{I}_{j i}
$$

where $\delta_{i j}$ is Kronecker's delta and

$$
I_{j j}\left([\mathbf{j}] \mid\left[\mathbf{j}^{\prime}\right]\right)=\sum_{P\left[\mathbf{j}^{\prime}\right]} \delta\left([\mathbf{j}]-\left[\mathbf{j}^{\prime}\right]\right)
$$

Since the components of vectors are symmetric functions of the particle phases, we have symmetrized the kernels (3.8) by inserting a summation over all permutations $P\left[\mathrm{j}^{\prime}\right]$ of the set $\left[\mathrm{j}^{\prime}\right]$. For example,

$$
I_{22}\left([\mathbf{2}] \mid\left[\mathbf{2}^{\prime}\right]\right)=\delta\left(\mathbf{x}_{1}-\mathbf{x}_{1}^{\prime}\right) \delta\left(\mathbf{x}_{2}-\mathbf{x}_{2}^{\prime}\right)+\delta\left(\mathbf{x}_{1}-\mathbf{x}_{2}^{\prime}\right) \delta\left(\mathbf{x}_{2}-\mathbf{x}_{1}^{\prime}\right)
$$

The structure of the reduced distribution functions associated with the probability density (3.1) can now be conveniently analyzed. We define two vectors $h=\left\{h_{k} ; k=1,2, \ldots\right\}$ and $\delta F=\left\{\delta F_{k} ; k=1,2, \ldots\right\}$, where $h_{k}$ are the group decomposition elements of $\rho$ and $\delta F_{k}=F_{k}-F_{k}^{\text {eq }}$ denotes the deviation of the $k$-particle reduced distribution from the equilibrium value $F_{k}^{\text {eq }}$. We get then the relation

$$
\delta F=\hat{Q} h
$$

with the operator $\hat{Q}$ defined by the following expression for the integral kernels:

$$
Q_{l i}\left([l] \mid\left[\mathbf{i}^{\prime}\right]\right)=\lim _{\infty}\left\langle\delta f([l] ; \Gamma) \delta f\left(\left[\mathbf{i}^{\prime}\right] ; \Gamma\right)\right\rangle, \quad l, i=1,2, \ldots
$$

where we use the notation $\langle\cdots\rangle \equiv\langle\cdots\rangle_{\rho^{\text {eq }}}$ and

$$
\delta f([l] ; \Gamma)=f([l] ; \Gamma)-\langle f([l] ; \Gamma)\rangle
$$

and $f([l] ; \Gamma)$ is defined by Eq. (2.6). A brief derivation of relation (3.10) is given in Appendix A.

We recall now an important decomposition of the operator $\hat{Q}$. The decomposition is crucial for our further considerations. Together with relation (3.10) it naturally leads to a new representation of the state of the system, which will be discussed in detail in the following section. as follows:

It has been shown in ref. II that the operator $\hat{Q}$ can be decomposed

$$
\hat{Q}=\hat{Q}^{(\beta)(\alpha)} \hat{Q}^{(\alpha)(\beta)} \hat{Q}
$$


where the operators at the right-hand side of Eq. (3.13) obey the conditions

$$
\begin{aligned}
& \hat{Q}_{i j}^{(\beta)}={ }^{(\beta)} \hat{Q}_{j i}=0 \quad \text { for } \quad j>i \\
& \hat{Q}_{i i}^{(\beta)}={ }^{(\beta)} \hat{Q}_{i i}=\hat{I}_{i i}
\end{aligned}
$$

and

$$
{ }^{(\alpha)} \hat{Q}_{i j}^{(\alpha)}=\delta_{i j}{ }^{(\alpha)} \hat{Q}_{i i}^{(\alpha)}
$$

The decomposition (3.13), together with supplementary conditions (3.14)(3.16), uniquely defines the operators $\hat{Q}^{(\beta)},{ }^{(\alpha)} \hat{Q}^{(\alpha)}$, and ${ }^{(\beta)} \hat{Q}$, which can be constructed by an iterative procedure. The details of the procedure along with explicit expressions for the kernels of the $\hat{Q}$-operators for small values of the indices $i$ and $j$ are given in II and will not be repeated here.

It can be seen from definition (3.11) that an integral kernel $Q_{k i}\left([\mathbf{k}] \mid\left[\mathbf{i}^{\prime}\right]\right)$ describes equilibrium correlations between two sets of particles $[\mathbf{k}]$ and $\left[\mathbf{i}^{\prime}\right]$. We recall the physical interpretation of decomposition (3.13) (3.16) of these correlations. This interpretation is supported by the virial-expansion analysis given in ref. $I$.

The kernel $Q_{k i}\left([\mathbf{k}] \mid\left[\mathbf{i}^{\prime}\right]\right)$ can be split into two parts: The first part describes all situations when there is an intermediate set of particles [p"] with $p \leqslant \min (k, i)$ if $k \neq i$ and with $p<i$ if $k=i$, such that (i) the particles $[\mathbf{k}]$ are correlated wih $\left[\mathbf{p}^{\prime \prime}\right]$, which in turn are correlated with $\left[\mathbf{i}^{\prime}\right]$; and (ii) there are no other correlations between $[\mathbf{k}]$ and $\left[\mathbf{i}^{\prime}\right]$. The second part of the kernel $Q_{k i}$ corresponds to the opposite situations, i.e., when no such intermediate set of particles can be found. We call these parts the reducible and irreducible correlations, respectively. In the above sense, the kernels of the operators $\hat{Q}_{k i}^{(\beta)},{ }^{(\alpha)} \hat{Q}_{i i}^{(\alpha)}$, and ${ }^{(\beta)} \hat{Q}_{k i}$ are the irreducible correlation functions for $k>i, k=i$, and $k<i$, respectively. Then Eq. (3.13) is the decomposition of complete correlations into irreducible ones.

We discuss now several important properties of the $Q$-operators. It follows immediately from definition (3.11) that the operator $\hat{Q}$ is self-adjoint.

$$
\hat{Q}^{\dagger}=\hat{Q}
$$

We also have the relations

$$
\left[\hat{Q}^{(\beta)}\right]^{\dagger}={ }^{(\beta)} \hat{Q}
$$

and

$$
\left[{ }^{(x)} \hat{Q}^{(\alpha)}\right]^{\dagger}={ }^{(x)} \hat{Q}^{(\alpha)}
$$

which result from the symmetry of the definition (3.13) (3.16). 
It was demonstrated in ref. II that the operators $\hat{Q}^{(\beta)}$ and ${ }^{(\beta)} \hat{Q}$ can be inverted. Indeed, since these operators have the form of triangular matrices with identity operators on the diagonal, explicit expressions for the matrix elements of the inverse operators can be easily constructed. Here we list only the expressions which are needed in our further considerations. It has been proven in ref. II that

$$
\left[\hat{Q}^{(\beta)}\right]_{i i}^{-1}=\left[{ }^{(\beta)} \hat{Q}\right]_{i i}^{-1}=\hat{I}_{i i}
$$

and

$$
\left[\hat{Q}^{(\beta)}\right]_{i j}^{-1}=\left[{ }^{(\beta)} \hat{Q}\right]_{j i}^{-1}=0 \quad \text { for } j>i
$$

We discuss also several important properties of the operator ${ }^{(\alpha)} \hat{Q}^{(\alpha)}$. It is shown in Appendix A that this operator is positive definite, i.e., for an arbitrary vector $H$, we have that

$$
\left(H,{ }^{(\alpha)} \hat{Q}^{(\alpha)} H\right) \geqslant 0
$$

The equal sign in the above relation holds if and only if $H=0$. Similar relations are fulfilled for all individual diagonal matrix elements ${ }^{(\alpha)} \hat{Q}_{k k}^{(\alpha)}$. Namely, for $k=1,2, \ldots$, we have the inequality

$$
\left(H_{k},{ }^{(\alpha)} \hat{Q}_{k k}^{(\alpha)} H_{k}\right) \geqslant 0
$$

with equal sign if and only if $H_{k}=0$.

We assume that the operator ${ }^{(\alpha)} \hat{Q}^{(\alpha)}$, as well as individual integral operators ${ }^{(\alpha)} \hat{Q}_{k k}^{(\alpha)}$, can be inverted. This assumption is based on the properties (3.22)-(3.23). Problems may occur only in extreme situations. For example, the Fourier transform of $n^{-1(\alpha)} \hat{Q}_{11}^{(\alpha)}$ is $S(k)$, the static structure factor, which for $k \rightarrow 0$ becomes the compressibility. In a closed-packed state this would be zero and therefore ${ }^{(\alpha)} \hat{Q}_{11}^{(\alpha)}$ could not be inverted. We want to exclude such situations from the domain of applicability of our results. A detailed analysis of the structure of the kernels of the operators inverse to ${ }^{(\alpha)} \hat{Q}_{k k}^{(\alpha)}$ is given in ref. 12. It is shown that the kernel of such an inverse operator can be expressed in terms of many-particle correlation functions which are natural generalizations of the Ornstein-Zernike direct paircorrelation function.

Finally, we introduce the notation

$$
\hat{Q}^{(\alpha)}=\hat{Q}^{(\beta)(\alpha)} \hat{Q}^{(\alpha)}
$$

and

$$
{ }^{(\alpha)} \hat{Q}=\left[\hat{Q}^{(\alpha)}\right]^{\dagger}={ }^{(\alpha)} \hat{Q}^{(\alpha)(\beta)} \hat{Q}
$$


We conclude from definitions (3.24)-(3.25) and the remarks above that the operators $\hat{Q}^{(x)}$ and ${ }^{(\alpha)} \hat{Q}$ can be inverted.

In the following section we analyze the consequences of the decomposition (3.13)-(3.16).

\section{ENSKOG-LIKE RENORMALIZATION}

The decomposition (3.13)-(3.16) can be conveniently used to define two new vectors $H$ and $\bar{H}$, each vector fully describing the statistical state of a system. It was demonstrated in refs. I and II that such a change of representation, at the level of the evolution equations, gives rise to an Enskog-like renormalization of the collisional events.

The vectors $H$ and $\bar{H}$ are related to a given set $h$ of cluster functions $h_{i}$ by the equations

and

$$
H={ }^{(\beta)} \hat{Q} h
$$

$$
\bar{H}={ }^{(\alpha)} \hat{Q} h
$$

Since Eq. (3.10), when supplemented with decomposition (3.13) and definitions (3.24)-(3.25), yields

$$
\begin{aligned}
\delta F & =\hat{Q}^{(\beta)} \bar{H} \\
& =\hat{Q}^{(\alpha)} H
\end{aligned}
$$

the vector $\bar{H}$ as well as $H$ entirely describes the nonequilibrium state of a system under consideration.

It follows from the above definitions and Eq. (3.25) that the vectors $H$ and $\bar{H}$ are related through the operator ${ }^{(\alpha)} \hat{Q}^{(\alpha)}$. Since the operator ${ }^{(\alpha)} \hat{Q}^{(\alpha)}$ has the diagonal structure (3.15), only components of $\bar{H}$ and $H$ with the same values of the indices are coupled, so that we have

$$
\bar{H}_{k}={ }^{(\alpha)} \hat{Q}_{k k}^{(\alpha)} H_{k}, \quad k=1,2, \ldots
$$

As we have noted in the preceding section the $\hat{Q}$-operators that relate the vectors $h, H, \bar{H}$, and $\delta F$ can be inverted. Therefore the representations of the state of a system in terms of these vectors are formally equivalent. However, the equivalence of the representations does not always hold on the level of individual components. This leads to very important differences between the representations if we consider an approximate description of the system, in terms of components with small values of the index only.

The pair $\bar{H}$ and $H$ is an exception in this regard. In this case, as we conclude from Eq. (4.5), for any $k$, individual components $\bar{H}_{k}$ and $H_{k}$ can 
be evaluated one from another and therefore include equivalent information. In particular, for any $i=1,2, \ldots$, approximate representations in terms of respective vectors $\bar{H}$ and $H$ with $\bar{H}_{k}$ and $H_{k}$ set equal to zero for all $k>i$ are equivalent.

We recall now the evolution equations for the vectors $\bar{H}$ and $H$ derived in I and II. First we introduce a convenient notation. Let $D$ be a binary-collision operator $\bar{T}_{ \pm}$or $T_{ \pm}$. We define a collisional operator $\hat{Q}[D]$ by the following equation:

$$
\left(Q_{i k}[D]\right)\left([\mathbf{i}] \mid\left[\mathbf{k}^{\prime}\right]\right)=\lim _{\infty}\left\langle\delta f([\mathbf{i}] ; \Gamma) D(\Gamma) \delta f\left(\left[\mathbf{k}^{\prime}\right] ; \Gamma\right)\right\rangle
$$

where

$$
D(\Gamma)=\sum_{i>j=1}^{N} D(i j)
$$

Next, we also define

$$
{ }^{(\alpha)} \hat{Q}^{(\beta)}[D]=\left[\hat{Q}^{(\beta)}\right]^{-1} \hat{Q}[D]\left[{ }^{(x)} \hat{Q}\right]^{-1}
$$

and

$$
{ }^{(\beta)} \hat{Q}^{(\alpha)}[D]=\left[\hat{Q}^{(\alpha)}\right]^{-1} \hat{Q}[D]\left[{ }^{(\beta)} \hat{Q}\right]^{-1}
$$

The operators ${ }^{(\alpha)} \hat{Q}^{(\beta)}[D]$ and ${ }^{(\beta)} \hat{Q}^{(\alpha)}[D]$ describe binary collisions renormalized by irreducible equilibrium correlations. They appear in the evolution equations for the vectors $\bar{H}$ and $H$. Explicit expressions for the elements of the operators ${ }^{(\alpha)} \hat{Q}^{(\beta)}[D]$ and ${ }^{(\beta)} \hat{Q}^{(\alpha)}[D]$, along with some other important results, were given in ref. II. In particular it has been shown there that

$$
{ }^{(a)} \hat{Q}_{k r}^{(b)}\left[\bar{T}_{\mp}\right]={ }^{(a)} \hat{Q}_{r k}^{(b)}\left[T_{\mp}\right]=0 \quad \text { for } \quad r>k+1
$$

where $(a, b)=(\alpha, \beta)$ or $(\beta, \alpha)$.

With the help of the above definitions the BBGKY hierarchy (2.8) can be transformed into hierarchies of evolution equations for the components of the vectors $\bar{H}$ and $H$. A detailed derivation of the hierarchies was given in ref. II. We list here only the final results. The evolution equations for the vector $\bar{H}$, expressed for individual components, for positive times, form the following hierarchy:

$$
\begin{aligned}
\left\{\partial_{t}+\hat{L}_{0, k}\right\} \bar{H}_{k}= & { }^{(\alpha)} \hat{Q}_{k, k-1}^{(\beta)}\left[T_{-}\right] \bar{H}_{k-1}+{ }^{(\alpha)} \hat{Q}_{k, k}^{(\beta)}\left[\bar{T}_{-}\right] \bar{H}_{k} \\
& +{ }^{(\alpha)} \hat{Q}_{k, k+1}^{(\beta)}\left[\bar{T}_{-}\right] \bar{H}_{k+1}, \quad k=1,2, \ldots
\end{aligned}
$$


Note the operator $T_{-}$that appears in the subdiagonal elements of the operator at the right-hand side of the above equations. This should be contrasted with the BBGKY hierarchy (2.8), where only the operators $\bar{T}_{-}$ appear. The operator $T_{-}$appears in Eq. (4.11) due to a commutation of the equilibrium correlations in the kernels of the operator $\hat{Q}^{(\beta)}$ with the $\hat{L}_{0}$ operator present at the left-hand side of (2.8).

Note also the tridiagonal structure of the hierarchy (4.11) that results from the property (4.10).

The evolution equations for the components of the vector $H$, for $t>0$, have a similar form. Namely, we have that

$$
\begin{aligned}
\left\{\partial_{\imath}+\hat{L}_{0, k}\right\} H_{k}= & { }^{(\beta)} \hat{Q}_{k, k-1}^{(\alpha)}\left[T_{-}\right] H_{k-1}+{ }^{(\beta)} \hat{Q}_{k, k}^{(\alpha)}\left[T_{-}\right] H_{k} \\
& +{ }^{(\beta)} \hat{Q}_{k, k+1}^{(\alpha)}\left[\bar{T}_{-}\right] H_{k+1}, \quad k=1,2, \ldots
\end{aligned}
$$

In the above equation the $T_{-}$operator appears in the subdiagonal and diagonal matrix elements.

As with the BBGKY hierarchy (2.8), the hierarchies (4.11) and (4.12), with operators $\bar{T}_{-}$and $T_{-}$, should be used for positive times only. For negative times, $t<0$, one should replace $\bar{T}_{-}$and $T_{-}$with the respective operators $-\bar{T}_{+}$and $-T_{+}$.

Hierarchies (4.11) and (4.12) are formally equivalent to the BBGKY hierarchy (2.8). However, by the transformation we have achieved a very important goal: The collisional operators $\bar{T}$ and $T$ are now renormalized by equilibrium correlations described by $Q$. Such renormalizations are of great importance if we consider approximate descriptions of systems in terms of functions depending on a small number of particle phases only. The renormalization of binary collisions achieved in Eqs. (4.11) and (4.12) is a generalization of the ideas at the basis of the Enskog equation.

We note that not all binary-collision operators in the hierarchies (4.11) and (4.12) are renormalized. In the case of (4.12) the detailed calculations presented in ref. II lead to the conclusion that

$$
{ }^{(\beta)} \hat{Q}_{k+1, k}^{(\alpha)}\left[T_{ \pm}\right]=\hat{I}_{k+1, k}\left[T_{ \pm}\right]
$$

where the operator $\hat{I}_{k+1, k}\left[T_{ \pm}\right]$is defined by the equation

$$
\hat{I}_{k+1, k}\left[T_{ \pm}\right]=\hat{I}_{k+1, k+1} \sum_{i=1}^{k} T_{ \pm}(i, k+1)
$$

The operator $\hat{I}_{k+1, k+1}$, defined in Eq. (3.8), has been introduced here to symmetrize the result of the action of the collisional operator. We can see from Eqs. (4.13)-(4.14) that the operator ${ }^{(\beta)} \hat{Q}_{k+1, k}^{(\alpha)}\left[T_{ \pm}\right]$does not contain any equilibrium correlations. One can show that all the remaining collisional 
operators appearing in the hierarchy (4.12) are, however, renormalized. Similar results hold also in the case of the hierarchy (4.11), where, in turn, the operator ${ }^{(\alpha)} \hat{Q}_{k, k+1}^{(\beta)}\left[\bar{T}_{ \pm}\right]$does not contain equilibrium correlations. The explicit form (4.13) of the collisional operator will be needed in our further considerations.

It is interesting to consider a sequence of approximations obtained by truncating the hierarchy (4.11) or (4.12). The $i$ th truncation is obtained, for $i=1,2, \ldots$, by setting $\bar{H}_{k}=0\left(H_{k}=0\right)$ for all $k>i$.

First of all we notice that the approximate kinetic equations obtained by the corresponding truncations made in $\bar{H}$ and $H$ representations are equivalent. To see this, we recall that the approximate representations of the state of a system in terms of the truncated vectors $\bar{H}$ and $H$ that have their components related by Eq. (4.5) are equivalent. [See comment below Eq. (4.5).] Our approximations are obtained by inserting such equivalent approximate representations into the exact evolution equations (4.11) and (4.12), so that the resulting time derivatives of the truncated vectors $\bar{H}$ and $H$ must be also equivalent.

It has been shown in ref. II that in the simplest case, i.e., when we truncate the hierarchies at the one-particle level, one gets the LREE. This convinces us that the renormalization of the collisional operators in Eqs. (4.11)-(4.12) is Enskog-like. The second truncation, with $i=2$, gives the LREE supplemented with terms corresponding to the renormalized ring and repeated ring dynamical events. Both of the equations mentioned above are fundamental for the theory of a dense hard-sphere fluid.

In the following section we consider the general truncation scheme. We show that the approximate kinetic equations obtained by such truncations all imply an irreversible approach to equilibrium of a hard-sphere system. To show this, we define, for cach kinetic equation associated with a given level of approximation, an entropy functional (or $H$-function). We then prove that the entropy functional monotonically increases in time, toward its equilibrium value. We also demonstrate that the equilibrium states are the only stationary solutions of the truncated hierarchy.

In particular, on the simplest level of approximation, with $i=1$, we recover in this way the $H$-theorem for the LREE that has been proven previously by Résibois. ${ }^{(5)}$ The $H$-theorems obtained for $i>1$ are new.

\section{APPROACH TO EQUILIBRIUM}

Before going on to discuss the $H$-theorems for the truncated hierarchies (4.11) and (4.12), we first consider some general properties of the entropy functional (1.4). For a system slightly displaced from equilibrium, with a 
distribution $\rho$ of the form (3.1)-(3.2), in the thermodynamic limit, to dominant order in the deviation from equilibrium we have that

$$
\delta S=S[\rho]-S\left[\rho^{\mathrm{eq}}\right]=-\frac{k_{\mathrm{B}}}{2} \lim _{\infty}\left\langle[\delta \psi(\Gamma)]^{2}\right\rangle
$$

where $\delta \psi=\psi-\langle\psi\rangle$. For simplicity we have assumed here that the considered deviation of $\rho$ from equilibrium preserves the number of particles, the momentum, and the energy, that is,

$$
\int d \Gamma \sum_{i=1}^{N} \chi_{a}(i)\left[\rho(\Gamma)-\rho^{\mathrm{eq}}(\Gamma)\right]=0
$$

where $\chi_{a}(v), a=1, \ldots, 5$ is equal, respectively, to $1, v_{x}, v_{v}, v_{z}, v^{2}$. We assume that in the thermodynamic limit the entropy functional $\delta S$ remains finite, which is possible if the deviation is localized in space.

The entropy functional $\delta S$ can be conveniently expressed by the vectors $H$ and $\bar{H}$. Namely, from Eqs. (5.1), (A.2), and (A.6) we get

$$
\delta S=-\frac{k_{\mathrm{B}}}{2}(H, \bar{H})
$$

As it follows from the definition of the scalar product in our vector notation, the entropy functional $\delta S$ is an infinite sum over $k$-particle terms

$$
\delta S=\sum_{k=1}^{\infty} \delta S_{k}
$$

with

$$
\delta S_{k}=-\frac{k_{\mathrm{B}}}{2}\left(H_{k}, \bar{H}_{k}\right)
$$

It is clear from Eq. (5.1) that $\delta S$ is bounded from above by zero, namely

$$
\delta S \leqslant 0
$$

where the equal sign holds if $\psi=0$ only. Also, one can see from inequality (3.23) and the relation (4.5) between $H_{k}$ and $\bar{H}_{k}$ that all components $\delta S_{k}$ are nonpositive

$$
\delta S_{k} \leqslant 0, \quad k=1,2, \ldots
$$

It is well known that the exact entropy functional (1.4) does not change under the exact time evolution of $\rho(t)$. This property is preserved 
if we retain the leading term alone in the expansion around equilibrium, as in Eq. (5.1). One can easily check using the time invariance of the equilibrium distribution and the Liouville theorem that indeed

$$
\frac{d}{d t} \delta S(t)=0
$$

We will use this fact to prove the $H$-theorem for the hierarchies (4.11) and (4.12) truncated by setting the components $\bar{H}_{k}^{(i)}$ and $H_{k}^{(i)}$ equal to zero for all $k>i$. We will show that for a system described by the truncated hierarchies the corresponding truncated entropy functional $\delta S^{(i)}$, defined by the equation

$$
\delta S^{(i)}=\sum_{k=1}^{i} \delta S_{k} \leqslant 0
$$

is a nondecreasing function of time,

$$
\frac{d}{d t} \delta S^{(i)} \geqslant 0
$$

for all $t \geqslant 0$, and find the states for which

$$
\frac{d}{d t} \delta S^{(i)}=0
$$

Using the $H$-theorem, one can argue in a standard way that the truncated hierarchies describe the approach of the system toward equilibrium.

The inequality (5.10) can be demonstrated in the following way: At any arbitrarily chosen time $t_{0} \geqslant 0$ we consider the solution $H(t)$ of the entire hierarchy (4.12) with the initial condition

$$
H\left(t_{0}\right)=H^{(i)}\left(t_{0}\right)
$$

One can see then that at $t=t_{0}$

$$
\partial_{t} H_{k}(t)=\partial_{t} H_{k}^{(i)}(t) \quad \text { for } \quad k=1, \ldots, i
$$

and

$$
\partial_{t} H_{k}(t)=0 \quad \text { for } \quad k>i+1
$$

It follows from Eq. (5.13) that at $t=t_{0}$ one can identify the time derivatives $d\left(\delta S^{(i)}\right) / d t$ calculated from the full and from the truncated hierarchies. Moreover, due to (5.14), for the full hierarchy we have that

$$
\frac{d}{d t} \delta S(t)=\frac{d}{d t} \delta S^{(i)}(t)+\frac{d}{d t} \delta S_{i+1}(t), \quad t=t_{0}
$$


But we know that the exact entropy functional $\delta S(t)$ does not change in time. Therefore we get the following relation:

$$
\frac{d}{d t} \delta S^{(i)}(t)=-\frac{d}{d t} \delta S_{i+1}(t), \quad t=t_{0}
$$

Since, according to our assumption, $\delta S_{i+1}\left(t_{0}\right)=0$, inequality (5.10) follows now immediately from (5.7) and Eq. (5.16).

To find the states for which the entropy functional $\delta S^{(i)}$ assumes its stationary value, we choose the following way. First we note that, since (5.16) holds, it is sufficient to consider, at any time $t=t_{0}$, the time derivative $d\left(\delta S_{i+1}\right) / d t$ calculated from the full hierarchy with the initial condition (5.12). In this way, by taking into account Eq. (5.5) and the relation (4.5) between the vectors $H$ and $\bar{H}$, we get the following equation:

$$
\frac{d}{d t} \delta S^{(i)}=-\frac{k_{\mathrm{B}}}{2} \frac{d}{d t}\left(H_{i+1},{ }^{(\alpha)} \hat{Q}_{i+1, i+1}^{(\alpha)} H_{i+1}\right), \quad t=t_{0}
$$

where

$$
H_{i+1}\left(t_{0}^{-}\right)=0
$$

and

$$
\left\{\partial t+\hat{L}_{0, i+1}\right\} H_{i+1}(t)=\hat{I}_{i+1, i}\left[T_{-}\right] H_{i}(t), \quad t \geqslant t_{0}
$$

Equation (5.19) is obtained from the hierarchy equation (4.12) by setting $k=i+1$, and neglecting at the right-hand side the two last collisional terms. According to our construction, for all $l>i$ we have that $H_{l}\left(t_{0}^{-}\right)=0$ and therefore the neglected collisional terms in Eq. (5.19) do not contribute to the time derivative in $\mathrm{Eq}$. (5.17). In a derivation of (5.19) expression (4.13) for the ${ }^{(\beta)} \hat{Q}_{i+1, i}^{(\alpha)}\left[T_{-}\right]$operator has been also taken into account.

The evaluation of the time derivative at the right-hand side of Eq. (5.17) requires a careful treatment of the discontinuity of the function $H_{i+1}$ that appears for $t>t_{0}$ due to the singular hard-core interactions. Since the calculations are long, part of them are relegated to Appendix B and we present here only the final steps of the analysis.

As a result of the detailed analysis presented in Appendix B, we conclude that

$$
\frac{d}{d t} \delta S^{(i)}(t)=0
$$


if and only if

$$
\hat{I}_{i+1, i}\left[T_{-}\right] H_{i}^{(i)}(t)=0
$$

By taking into account the explicit form (B.1)-(B.2) of the operator $\hat{I}_{i+1, i}\left[T_{-}\right]$, we find that Eq. (5.21) is equivalent to

$$
\delta\left(r_{i, i+1}-\sigma^{+}\right) \theta\left(\mathbf{v}_{i, i+1} \cdot \hat{\mathbf{r}}_{i, i+1}\right)[\hat{b}(i, i+1)-1]\left[1+P_{i, i+1}\right] H_{i}^{(i)}([\mathbf{i}] ; t)=0
$$

where the operator $P_{i, i+1}$ permutes the particles $i$ and $i+1$.

Here we can use the results obtained by Résibois ${ }^{(5,6)}$ in his papers on the $H$-theorem for the REE. He considered there an equation analogous to (5.22), but with a function $H$ depending on a single-particle phase only. However, we can use here his results if we simply treat the phases of the particles $1, \ldots, i-1$ as parameters. By adopting Résibois' result we conclude that a solution of Eq. (5.22) has the following form:

$$
H_{i}^{(i)}([\mathbf{i}] ; t)=f\left([\mathbf{i}-\mathbf{1}], \mathbf{r}_{i}\right)+\sum_{a=1}^{5} g_{a}([\mathbf{i}-\mathbf{1}]) \chi_{a}\left(\mathbf{v}_{i}\right)
$$

where $\chi_{a}(\mathbf{v})$ are the additive collisional invariants and $f$ and $g_{a}$ are arbitrary functions.

We assumed that the entropy functional $\delta S^{(i)}$ is finite, which property, due to inequalities (5.9)-(5.10), is preserved in time. For states with finite $\delta S^{(i)}$ the function $H_{i}^{(i)}$ tends to 0 when $r_{i} \rightarrow \infty$. Therefore all the functions $g_{a}$ have to vanish. Next, since $H_{i}^{(i)}$ is a symmetric function of phases of particles, one can see from Eq. (5.23) that $H_{i}^{(i)}$ can depend on the positions of particles only, so that we have

$$
H_{i}^{(i)}([\mathbf{i}] ; t)=H_{i}^{(i)}\left(\mathbf{r}_{1}, \ldots, \mathbf{r}_{i}\right)
$$

This is a sufficient and necessary condition on the vector $H^{(i)}$ for the entropy functional $\delta S^{(i)}$ to assume a stationary value at a time $t$.

To complete the proof of the $H$-theorem, we should find all stationary solutions of the truncated hierarchy (4.12). Equation (5.20), and therefore also (5.24), obviously is a necessary condition. The $i$ th equation of the truncated hierarchy (4.12) with $H_{i}^{(i)}$ given in the form (5.24) is then equivalent to the following condition:

$$
\hat{L}_{0, i} H_{i}^{(i)}=\hat{I}_{i, i-1}\left[T_{-}\right] H_{i-1}^{(i)}
$$

One can see from the expression (4.14) for the operator $\hat{I}_{i, i-1}\left[T_{-}\right]$that due to the factor $\theta\left(-\mathbf{v}_{k l} \cdot \hat{\mathbf{r}}_{k l}\right)$ that appears in the definition $(2.1)$ of the 
operator $T_{-}$the right-hand side of the above equation vanishes for particle velocities such that $\mathbf{v}_{k l} \cdot \hat{\mathbf{r}}_{k l}>0$ for all pairs with $k, l=1, \ldots, i$. Thus, since a stationary $H_{i}^{(i)}$ depends only on particle positions, Eq. (5.25) can be fulfilled only if $H_{i}^{(i)}$ is constant. For states with a finite entropy, $H_{i}^{(i)}$ vanishes at infinity and therefore condition (5.25) can be fulfilled only if

$$
H_{i}^{(i)}(t)=0
$$

Using this result, from (5.25) we obtain again Eq. (5.21), but now with the subscripts $i+1, i$ replaced with $i, i-1$. Therefore, we can repeat the arguments and show that $H_{k}^{(i)}=0$ for all $k=1, \ldots, i$. As a result, we find that the only stationary solution of the truncated hierarchy (4.12) is the vector

$$
H^{(i)}=0
$$

Using similar arguments, one can show that a solution $H^{(i)} \neq 0$ of the truncated hierarchy cannot remain, over a finite interval of time, in a subspace of vectors for which $\delta S$ assumes a stationary value.

The above results can be used to argue, in a standard way, that the truncated hierarchy describes the approach of a hard-sphere system toward equilibrium. Namely, since $\delta S(t)$ is an increasing function bounded from above, it approaches a stationary value when time goes to infinity. One usually assumes that this can happen only if the solution of the hierarchy itself also approaches a stationary value. Since the only stationary solution of the hierarchy is the equilibrium solution $H^{(i)}=0$, we conclude then that the system approaches equilibrium. A rigorous proof that solutions of a kinetic equation do approach the equilibrium value requires, even for a linear theory, a more detailed analysis of the structure of the collisional operators.

\section{TIME-CORRELATION FUNCTIONS}

A very important application of the LKT is the calculation of equilibrium time-correlation functions. In this section we briefly discuss some aspects of this application. In particular, we show that the symmetry properties of time-correlation functions that reflect the symmetry of evolution equations under reversal of time and particle velocities are preserved when the truncated hierarchies (4.11) and (4.12) are used to approximately describe the evolution of a hard-sphere system. Indeed, one expects that the symmetries are preserved on the approximate level of description since the truncations are obtained from the exact evolution equations by using closure approximations that are manifestly symmetric with respect to time or velocity reversal. 
The time-correlation function for two observables $A(\Gamma)$ and $B(\Gamma)$ can be defined in the following way:

$$
G_{A B}( \pm t)=\lim _{\infty} \int d \Gamma \delta A^{*}(\Gamma) \delta B(\Gamma(\mp t)) \rho^{\mathrm{eq}}(\Gamma), \quad t \geqslant 0
$$

The interest lies almost exclusively, in time-correlation functions between $A$ and $B$ that both have a linear group decomposition of the form (A.1). In such cases, to describe the time-correlation functions one can naturally apply the LKT formalism. Usually, in physically interesting cases, only the first and possibly the second term in the group decomposition of $A$ and $B$ differs from zero.

For hard spheres, the problem of calculating the time-correlation function (6.1) may be formulated in the following way. First one should find the "distribution" $\delta \rho^{B}(\Gamma ; \pm t)$ that solves the pseudo-Liouville equation

$$
\partial_{t} \delta \rho^{B}(\Gamma ; \pm t)=\mp \bar{L}_{\mp}(\Gamma) \delta \rho^{B}(\Gamma ; \pm t)
$$

with the initial condition

$$
\delta \rho^{B}(\Gamma ; 0)=\delta B(\Gamma) \rho^{\mathrm{eq}}(\Gamma)
$$

Next we calculate the "average"

$$
G_{A B}( \pm t)=\int d \Gamma \delta A^{*}(\Gamma) \delta \rho^{B}(\Gamma ; \pm t)
$$

By $\bar{L}_{ \pm}(\Gamma)$ we denote the hard-sphere pseudo-Liouville operator, defined by the equation

$$
\bar{L}_{ \pm}(\Gamma)=\hat{L}_{0}(\Gamma) \pm \sum_{k>l=1}^{N} \bar{T}_{ \pm}(i j)
$$

Initial condition (6.3), supplemented with the group decomposition of the function $B$, is consistent with the basic assumption of the LKT, formulated in Eqs. (3.1)-(3.2). We can thus use, in this context, the formalism described in Sections 3 and 4.

To this end, we first calculate from $\delta \rho^{B}( \pm t)$ the reduced distribution functions $\delta F_{B, k}( \pm t)$. In the next step, we apply the transformation 'described in detail in Section 4 to obtain two equivalent representations $\bar{H}_{B}( \pm t)$ and $H_{B}( \pm t)$, related to the set of reduced distribution functions by equations analogous to (4.3) and (4.4). The evolution of the vectors $\bar{H}_{B}( \pm t)$ and $H_{B}( \pm t)$ is governed by the respective hierarchies (4.11) and (4.12), with proper modification in the case of negative times. 
Let us denote by $\overline{\mathscr{L}}^{ \pm}$and $\mathscr{L}^{ \pm}$the linear operators that generate the evolution of the vectors $\bar{H}( \pm t)$ and $H( \pm t)$. According to Eqs. (4.11) and (4.12), the matrix elements of these operators are

$$
\begin{aligned}
\overline{\mathscr{L}}_{k l}^{ \pm}= & \delta_{k l}\left\{\hat{L}_{0, k} \pm{ }^{(\alpha)} \hat{Q}_{k k}^{(\beta)}\left[\bar{T}_{ \pm}\right]\right\} \pm \delta_{k+1, l}{ }^{(\alpha)} \hat{Q}_{k, k+1}^{(\beta)}\left[\bar{T}_{ \pm}\right] \\
& \pm \delta_{k-1, l}{ }^{(\alpha)} \hat{Q}_{k, k-1}^{(\beta)}\left[T_{ \pm}\right]
\end{aligned}
$$

and

$$
\begin{aligned}
\mathscr{L}_{k l}^{ \pm}= & \delta_{k l}\left\{\hat{L}_{0, k} \pm{ }^{(\beta)} \hat{Q}_{k k}^{(\alpha)}\left[T_{ \pm}\right]\right\} \pm \delta_{k+1, l}{ }^{(\beta)} \hat{Q}_{k, k+1}^{(\alpha)}\left[\bar{T}_{ \pm}\right] \\
& \pm \delta_{k-1, l}{ }^{(\beta)} \hat{Q}_{k, k-1}^{(\alpha)}\left[T_{ \pm}\right]
\end{aligned}
$$

With this notation, we can write the solutions of the hierarchies (4.11) and (4.12) in the form

$$
\bar{H}_{B}( \pm t)=\exp \left[\mp \overline{\mathscr{L}}^{\mp} t\right] \bar{H}_{B}
$$

and

$$
H_{B}( \pm t)=\exp \left[\mp \mathscr{L}^{\mp} t\right] H_{B}
$$

where $H_{B}$ and $\bar{H}_{B}$ denote the initial conditions at time $t=0$. The vectors $H_{B}$ and $\bar{H}_{B}$ are related to the group decomposition elements $h_{B}$ of the function $B$ by relations analogous to (4.1)-(4.2).

The time-correlation function $G( \pm t)$ can be conveniently expressed in terms of the $H$-vectors. Using Eqs. (6.8)-(6.9) and the results of Appendix A, we get the equations

$$
\begin{aligned}
G_{A B}( \pm t) & =\left(H_{A}, \exp \left[\mp \overline{\mathscr{L}}^{\mp} t\right] \bar{H}_{B}\right) \\
& =\left(\bar{H}_{A}, \exp \left[\mp \mathscr{L}^{\mp} t\right] H_{B}\right)
\end{aligned}
$$

The above expressions are a convenient starting point to discuss some important symmetry properties of time-correlation functions.

The first symmetry property considered is the following:

$$
G_{A B}(t)=G_{B A}(-t)
$$

It results from the definition (6.1) of the time-correlation function, the Liouville theorem, and the time invariance of the equilibrium distribution function. The next important symmetry, which is a consequence of the invariance of the pseudo-Liouville equation with respect to simultaneous inversion of time and velocity, can be expressed as follows:

$$
G_{A B}(t)=G_{\hat{p} A, \hat{p} B}(-t)
$$


where the operator $\hat{p}$ changes the variables $\mathbf{v}_{i}$ into $-\mathbf{v}_{i}$ for all $i=1,2, \ldots$ (We may consider here the inversion of the spatial variables $\mathbf{r}_{i}$ instead; the Liouville operator is invariant under inversion of the particle phases and therefore the inversions of the velocities and the space coordinates are equivalent in the present context.) The symmetries (6.12) and (6.13), taken together, result in the important symmetry property

$$
G_{A B}(t)=G_{p B, \hat{p} A}(t)
$$

On the level of a renormalized desciption of the system, in terms of the vectors $\bar{H}$ and $H$, the symmetry (6.12) can be related to the following property of the operator $\overline{\mathscr{L}}^{ \pm}$:

$$
\left[\overline{\mathscr{L}}^{ \pm}\right]^{\dagger}=-\mathscr{L}^{\mp}
$$

This property is a direct consequence of the definitions (6.6)-(6.7) and the symmetry properties of the $Q$-operators, derived in ref. II. To relate the symmetry (6.12) of the time-correlation function to the property (6.15) of the renormalized Liouville operator $\mathscr{L}$, we note that from (6.15) we get the relation

$$
\left(H_{A}, \exp \left[\overline{\mathscr{L}}^{+} t\right] \bar{H}_{B}\right)=\left(\exp \left[-\mathscr{L}^{-} t\right] H_{A}, \bar{H}_{B}\right)
$$

and that the representations $(6.10)$ and $(6.11)$ of the time-correlation function are equivalent.

The next symmetry property of the time-correlation functions, given in Eq. (6.13), is related, in a similar way, to the following property of the operators $\mathscr{L}_{ \pm}$:

$$
\hat{p} \mathscr{L}^{+} \hat{p}=-\mathscr{L}^{-}
$$

The above relation is a consequence of the definition (6.7), the relation

$$
\hat{p} T_{+} \hat{p}=-T_{-}
$$

and the fact that the equilibrium distributions included in the kernels of the $Q$-operators are invariant under the inversion of velocities. [To obtain the symmetry (6.13), one can also use a relation for the $\overline{\mathscr{L}}$-operators that is analogous to $(6.17)$.]

Let us now consider the $i$ th truncation of the hierarchies (4.11) and (4.12). We can easily argue that the symmetry properties (6.12)-(6.13) of the time-correlation functions-and therefore also the symmetry (6.14) remain valid at the approximate level of description. To show this, we simply notice that the symmetries (6.15) and (6.17) hold not only for the full $\mathscr{L}$-operators, but also for the truncated ones, with the matrix elements 
$\overline{\mathscr{L}}_{k l}^{ \pm}$and $\mathscr{L}_{k l}^{ \pm}$set equal to zero if $k$ or $l>i$. Taking this into account, together with the equivalence of the $i$ th truncations of the hierarchies (4.11) and (4.12), one can repeat the arguments given above for the case of the exact hierarchies and show that the symmetry properties (6.12)-(6.14) hold also if time-correlation functions are evaluated approximately by using the truncated hierarchies.

We recall that, within our approximation scheme, the LREE is obtained as a special case by truncating on the one-particle level. Therefore, at this level, we recover here the symmetry properties of the linearized Enskog operator, which have been extensively studied in the literature. ${ }^{(13-15)}$

\section{DISCUSSION}

For the approximate LKT derived by Cichocki and Bławzdziewicz a strong $H$-theorem could be proved: Entropy is strictly increasing in time, unless the $i$ th reduced distribution function depends on position coordinates only. Although it follows that the only stationary solution of the LKT is the equilibrium solution, this does not suffice to prove the asymptotic approach of the reduced distribution functions to zero for long times.

In addition, we showed that the usual symmetry properties of equilibrium time-correlation functions are preserved under truncations of the hierarchy, as employed in the LKT of Cichocki and Blawzdziewicz.

One may speculate that application of the maximization of entropy principle $^{(2,4)}$ at the level of the $i$-particle distribution function $f([\mathbf{i}])$ will give rise to nonlinear kinetic equations for which, in the case of hard spheres, a strong $H$-theorem can be proved. One expects that under linearization these kinetic equations would reduce to the LKT of Bławzdziewicz and Cichocki. In fact, the LKT of Blawzdziewicz and Cichocki can be derived straightforwardly using the maximization of entropy principle applied to the approximate entropy functional (5.1).

\section{APPENDIX A}

In this appendix we derive Eqs. (3.10) and (3.22)-(3.23) and some other important relations for the $Q$-operators introduced in Section 3 . We start by analyzing the static structure of equilibrium correlations.

Consider two phase-space functions $A(\Gamma)$ and $B(\Gamma)$, both with a group decomposition analogous to (3.2), so that we have

$$
a(\Gamma)=\sum_{i=1}^{N} h_{a, 1}\left(\mathbf{x}_{i}\right)+\sum_{i>j=1}^{N} h_{a, 2}\left(\mathbf{x}_{i}, \mathbf{x}_{j}\right)+\sum_{i>j>k=1}^{N} h_{a, 3}\left(\mathbf{x}_{i}, \mathbf{x}_{j}, \mathbf{x}_{k}\right)+\cdots
$$


where $a=A, B$. The equilibrium correlation function between $A$ and $B$ is defined by

$$
G_{A B}=\lim _{\infty}\left\langle\delta A^{*}(\Gamma) \delta B(\Gamma)\right\rangle
$$

By inserting into Eq. (A.2) the functions $A$ and $B$ expressed in the form

$$
a(\Gamma)=\sum_{i=1}^{N} \frac{1}{i !} \int d[\mathbf{i}] f([\mathbf{i}] ; \Gamma) h_{a, i}([\mathbf{i}])
$$

where $a=A, B$ and $f([\mathbf{i}] ; \Gamma)$ is defined in Eq. (2.6), and then using the definitions (3.11) and (3.3) (3.6), one gets

$$
G_{A B}=\left(h_{A}, \hat{Q} h_{B}\right)
$$

Equation (3.10) can be obtained in a similar way, by using definition (2.5) and Eqs. (3.1)-(3.2).

The correlation function $G_{A B}$ can be expressed in terms of the $H$-vectors, defined by Eqs. (4.1) and (4.2) (with $h=h_{A}$ or $h_{B}$ ). Namely, taking into account decomposition (3.13) and the property (3.18), one gets

$$
\begin{aligned}
G_{A B} & =\left(H_{A},{ }^{(\alpha)} \hat{Q}^{(\alpha)} H_{B}\right) \\
& =\left(H_{A}, \bar{H}_{B}\right)
\end{aligned}
$$

It is obvious from definition (A.2) that for any $A$

$$
G_{A A} \geqslant 0
$$

Hereafter we identify all phase-space functions which differ for overlapping configurations only. With this convention applied, we conclude from (A.2) that $G_{A A}=0$ if and only if $A=0$ or, equivalently, $h_{A}=0$. It was shown in ref. II that the operator ${ }^{(\beta)} \hat{Q}$ can be inverted. Therefore the equal sign in relation (A.7) holds if and only if $H_{A}=0$. From this analysis and relation (A.5) property $(3.22)$ of the ${ }^{(\alpha)} \hat{Q}^{(\alpha)}$ operator immediately follows. The property (3.23) of individual diagonal matrix elements can then be obtained by substituting into (3.22) a vector $H$ with the components $H_{i}=\delta_{i k} H_{k}$, where $i=1,2, \ldots$.

Expressions analogous to Eqs. (A.4)-(A.6) can also be obtained for a time correlation function $G_{A B}(t)$, as defined in Eq. (6.1). To obtain the representation for $G_{A B}(t)$ corresponding to Eq. (A.4), one should simply replace in this equation $h_{B}$ with $h_{B}(t)$, the vector of the group-decomposition elements of the distribution $\rho^{B}(t)$. [The distribution $\rho^{B}(t)$, associated with the time-correlation function, is defined by relations (6.2)-(6.3).] Relation 
(6.10) can then be obtained in a similar way as Eq. (A.6). Hence (6.11) is obtained by using the relation (4.5) between $\bar{H}$ and $H$-representations, together with the symmetry of the operator ${ }^{(x)} \hat{Q}^{(\alpha)}$.

\section{APPENDIX B}

In this appendix we demonstrate from Eqs. (5.17)-(5.19) that the entropy functional $\delta S^{(i)}$ assumes a stationary value if and only if condition (5.21) is satisfied. The main problem when calculating the time derivative of $\delta S^{(i)}$ from Eq. (5.17) is to correctly take into account the discontinuity of the function $H_{i+1}\left(t_{0}+\tau\right)$ that appears at $\tau \geqslant 0$ due to the singular character of the hard-sphere interaction.

We start by rewriting the operator $\hat{I}_{i+1, i}\left[T_{-}\right]$in a more explicit form. By taking into account Eq. (4.14) and definition (2.1) of the operator $T_{-}$, we arrive at the following expression:

$$
\hat{I}_{i+1, i}\left[T_{-}\right] H_{i}([\mathbf{i}] ; t)=\sum_{k>t=1}^{i+1} \delta\left(r_{k l}-\sigma^{+}\right) \omega^{(k l)}([\mathbf{i}+\mathbf{1}] ; t)
$$

where

$$
\omega^{(k l)}([\mathbf{i}+\mathbf{1}] ; t)=\theta\left(\mathbf{v}_{k l} \cdot \hat{\mathbf{r}}_{k l}\right)\left|\mathbf{v}_{k l} \cdot \hat{\mathbf{r}}_{k l}\right|[\hat{b}(k l)-1]\left(\hat{P}_{k, i+1}+\hat{P}_{l, i+1}\right) H_{i}([\mathbf{i}] ; t)
$$

and the operator $\hat{P}_{i j}$ permutes the phases of particles $i$ and $j$. The solution of Eqs. (5.18)-(5.19) has then the form

$$
H_{i+1}([\mathbf{i}+\mathbf{1}] ; t)=\int_{0}^{t-t_{0}} d \tau e^{-\tau \hat{L}_{0, i+1}} \sum_{k>l=1}^{i+1} \delta\left(r_{k l}-\sigma^{+}\right) \omega^{(k l)}\left([\mathbf{i}+\mathbf{1}] ; t_{0}+\tau\right)
$$

Assuming that $\omega^{(k l)}\left([\mathbf{i}+\mathbf{1}] ; t_{0}+\tau\right)$ is a sufficiently smooth function, up to terms of the order $\tau$, the solution (B.3) can be approximated by

$$
H_{i+1}\left([\mathbf{i}+1] ; t_{0}+\tau\right)=\sum_{k>l=1}^{i+1} \chi\left(\mathbf{x}_{k}, \mathbf{x}_{l} ; \tau\right) \omega^{(k l)}\left([\mathbf{i}+\mathbf{1}] ; t_{0}\right)+O(\tau)
$$

where

$$
\chi\left(\mathbf{x}_{k}, \mathbf{x}_{l} ; \tau\right)=\theta\left(r_{k l}+\left[\mathbf{v}_{k l} \cdot \mathbf{r}_{k l}\right] \tau-\sigma^{+}\right)-\theta\left(r_{k l}-\sigma^{+}\right)
$$

To obtain from Eq. (5.17) the time derivative of $\delta S^{(i)}$, it is sufficient to evaluate the linear term in $\tau$ in

$$
\delta S_{i+1}\left(t_{0}+\tau\right)=\left(H_{i+1}\left(t_{0}+\tau\right),{ }^{(\alpha)} \hat{Q}_{i+1, i+1}^{(\alpha)} H_{i+1}\left(t_{0}+\tau\right)\right)
$$


Let us insert (B.4) into the quadratic form (B.6). The linear contributions result only from those terms in (B.6) in which two factors $\chi\left(\mathbf{x}_{k}, \mathbf{x}_{l} ; \tau\right)$ with the same pair $(k l)$ of particle variables meet together. Taking this into account, one can show that

$$
\frac{d}{d t} \delta S_{i+1}=\sum_{k>l=1}^{i+1} \int d \mathbf{x}_{k} \int d \mathbf{x}_{l} \delta\left(r_{k l}-\sigma^{+}\right)\left(\omega^{(k l)}, \hat{A}_{i+1, i+1}^{(k l)} \omega^{(k l)}\right)_{k l}
$$

where the scalar product $\left(a^{(k l)}, b^{(k l)}\right)_{k l}$ of two functions $a^{(k l)}$ and $b^{(k l)}$ is defined by the equation

$$
\left(a^{(k l)}, b^{(k l)}\right)_{k l}=\frac{1}{(i+1) !} \int a^{(k l)}([\mathbf{i}+\mathbf{1}]) b^{(k l)}([\mathbf{i}+\mathbf{1}]) \hat{P}_{k, i} \hat{P}_{l, i+1} d[\mathbf{i}-\mathbf{1}]
$$

Here the variables $\mathbf{x}_{k}$ and $\mathbf{x}_{l}$ are not integrated over and are treated as parameters. The kernel of the operator $\hat{A}_{i+1, i+1}^{(k l)}$ is equal to the sum of all those terms contributing to ${ }^{(\alpha)} Q_{i+1, i+1}^{(\alpha)}\left([\mathbf{i}+\mathbf{1}] \mid\left[(\mathbf{i}+\mathbf{1})^{\prime}\right]\right)$ that include the product $\left(1+\hat{P}_{k l}\right) \delta\left(\mathbf{x}_{k}-\mathbf{x}_{k}^{\prime}\right) \delta\left(\mathbf{x}_{l}-\mathbf{x}_{l}^{\prime}\right)$.

Our proof can now be completed by noting that the operator $\hat{A}_{i+1, i+1}^{(k)}$ is positive definite with respect to the scalar product $(\cdot, \cdot)_{k l}$. This property can be demonstrated by adopting the arguments given in Appendix $\mathrm{A}$ for the case of the operator ${ }^{(\alpha)} \hat{Q}^{(\alpha)}$. Namely, the basic relations between $\hat{Q}$-operators, used in the proof, remain valid if the kernels of the operators are replaced by their respective parts that include the product $\left(1+\hat{P}_{k l}\right) \delta\left(\mathbf{x}_{k}-\mathbf{x}_{k}^{\prime}\right) \delta\left(\mathbf{x}_{l}-\mathbf{x}_{l}^{\prime}\right)$; by repeating the arguments of Appendix A (in inverse order), one can transform the scalar product at the rhs of Eq. (B.7) into the corresponding positive-definite ensemble average, with the variables $\mathbf{x}_{k}$ and $\mathbf{x}_{l}$ treated as parameters.

For $\hat{A}_{i+1, i+1}^{(k l)}$ positive definite, it immediately follows from (B.7) that $d\left(\delta S^{(i)}\right) / d t=0$ if and only if

$$
\delta\left(r_{k l}-\sigma^{+}\right) \omega^{(k l)}\left([\mathbf{i}+1] ; t_{0}\right)=0
$$

for all $k, l \leqslant i+1$ or, by taking into account relation (B.1), if and only if (5.21) holds.

\section{ACKNOWLEDGMENTS}

We acknowledge J. A. Leegwater for remarks on the manuscript. 


\section{REFERENCES}

1. J. Karkheck and G. Stell, Phys. Rev. A 25:3302 (1982).

2. J. Karkheck, H. van Beijeren, I. de Schepper, and G. Stell, Phys. Rev. A 32:2517 (1985).

3. J. Blawzdziewicz and G. Stell, J. Stat. Phys. 56:821 (1989).

4. H. van Beijeren, in Fundamental Problems in Statistical Mechanics, Vol. VII, H. van Beijeren, ed. (North-Holland, Amsterdam, 1990).

5. P. Résibois, Physica A 94:1 (1978).

6. P. Résibois, J. Stat. Phys. 19:593 (1978).

7. J. Bławzdziewicz and B. Cichocki, Physica A 127:38 (1984); referred to as I.

8. B. Cichocki, Physica A 142:245 (1987); referred to as II.

9. M. H. Ernst, J. R. Dorfman, W. R. Hogey, and J. M. J. van Leeuwen, Physica 45:127 (1969).

10. J. Piasecki, in Fundamental Problems in Statistical Mechanics, Vol. IV, E. G. D. Cohen and W. Fiszdon, eds. (Ossolineum, Wrocław, 1978).

11. H. van Beijeren, Kinetic theory of hard spheres, $\mathrm{Ph}$. D. thesis, University of Nijmegen (1974); H. van Beijeren and M. H. Ernst, J. Stat. Phys. 21:125 (1979).

12. J. Blawzdziewicz, B. Cichocki, and R. Hołyst, Physica A 157:857 (1989); R. Hołyst, J. Bławzdziewicz, and B. Cichocki, unpublished.

13. E. G. D. Cohen and I. M. de Schepper, J. Stat. Phys. 46:949 (1987).

14. G. Szamel, J. Stat. Phys. 55:381 (1989).

15. J. A. Leegwater, H. van Beijeren, and J. Michels, J. Phys. Condensed Matter 1:237 (1989). 